\title{
Society in Hand: Toward Community Service through Social Network
}

\author{
Abeer El-Korany \\ Computer Science Department \\ Faculty of Computers \& \\ Information, Cairo University \\ 5 Dr Ahmed Zoweil St., Orman, \\ Giza, Egypt
}

\begin{abstract}
Technology plays a significant role in the development of society. Social Networking Sites (SNS) have not only been used to cover the needs of entertainment, finding friends or family but also have been extended to cover other issues such as social and community services. This paper explores usefulness of using social network in the context of community and social services. By analyzing the linkage patterns among members of social network, communities of similar users are constructed. The proposed system, Society in hand, matches semantic of user's profiles in order to enable social workers, their colleagues, and others to keep connected, informed, and organized in form of community to support charity purposes. Using such a system would increase the effectiveness of charities activities and saves cost and time as well as would increase the participation of volunteers in community services.
\end{abstract}

\section{Keywords}

Social networks, knowledge sharing, ontology, similarity measure.

\section{INTRODUCTION}

Social networking is the process of linking of individuals into a set of groups according to common interest. Social networking is not only possible for person, especially in the workplace, universities, and schools, but also is most widespread as online site. Social software aims to enable people to connect or collaborate to form online communities by being able to accept or reject people into their network and communicate within them online, [6]. Online social networking in form of websites is commonly used. These websites are widely accepted as social sites. Social network websites are found as online communities of internet users. Depending on the scope of website, many of these online community members share common interests such as hobbies, religion, or politics. Once the users are allowed to access a social networking website they begin to socialize. This socialization may include accessing the profile pages of other members, sharing common knowledge, and possibly even contacting others [16]. Social network often involves grouping specific individuals or organizations with each other. Currently, there are a number of social networking websites that connect their members based on particular interests, there are others that do not. The websites without a main concern (interest) are often referred to as "traditional" social networking websites and usually its memberships is open for everyone. This means that anyone can become a member of this social network, no matter what their hobbies, beliefs, or views are. Some of these sites like MySpace, twitter, and Facebook were able to capture the attention of youth and changing the social landscape of college campuses and the teenagers. Another type of social network are those targeting a specific type of individual and aim to construct online communities of people that share common interests like for example ResearchGate, Labmeeting and others which are oriented to smaller market of today's modern researcher. People are great sources of information, especially that which is location-specific, community-specific and time-specific [14]. Therefore, social networking is effective in the community field because the type of information that need to be shared and not available anywhere else [14]. The aim of this paper is to present a framework that utilizes social network to facilitate the communication and collaborative work between different entities (individuals or organizations) that work in the social and community field. The proposed system, society in hand, facilitates the interaction between different types of people working in the community service field. Traditionally, charities and social associations used to provide to a set of services to community such as in-kind assistance, health care services, eradication of illiteracy, etc. Those services are accomplished through current employees who are working in the social association but sometimes it needs help from other entities. Society in hand enables volunteers to interact directly with social associations and with each other with an overall aim to deliver social and community services to all people. It extracts communities of volunteers based on the assumptions on that relationship among actors within a social network is determined based on their joint involvement in specific event which is expressed here as their tendency to apply specific set of community service. The main idea of clustering volunteers is to fulfill any request from any social associations when it requires volunteer(s) to handle a specific community service. Furthermore, the system regularly recommends suitable social associations for volunteers to deal with. The main functionality of the system could be summarized as:

- Provide a suitable platform to enable social associations and volunteers to interact and share information through appropriate information sharing environment;

- Track and learn semantic user preferences over time;

- Cluster volunteers into groups based on combined knowledge that decomposed explicit defined profiles and their dynamic interaction (analysis of their behavior) over time. Clustering of volunteers provides social associations the facility to select the suitable set of volunteers who are candidate to apply a specific service (s) whenever it asks for. Unlike other community social network such as Jumo.com, Given Tree , our system is capable of grouping similar volunteers in order to help them gain from each other's experience 
Furthermore, society in hand provides traditional social network services such as add friend, suggest friend, allow volunteer/charity to post their recent news and discuss, send message, etc. Society in hand is available as a web application with a friendly user interface in order to involve diverse citizens of the society to be a member in the social work and bridge the gap between different levels of community, allowing them to contribute and hence improve the community services delivery to slums. Moreover, it would contribute to enlargement of our charity network since it offers volunteers, appropriate channel through which they would carry out their effort. The structure of the paper is as follow, section2 describes different social networking types and analysis. Next, the approach of utilizing social network to improve knowledge sharing is introduced. Then, the general architecture of society in hand is presented in section 4 . The clustering process using ontology is described in section 5 and finally implantation of the system is presented in section6.

\section{BACKGROUND}

The proposed system is a social network that aims to facilitate interaction between individuals who are involved in community services. Social network sites could be classified into two main categories. The first category provides open memberships so that anyone can become a member, while the other category focus on particular interests such as research gate, ScienceStage, etc target a specific category of people to emphasis their activities. This section provides details about different types of social networks

\subsection{Social network}

A social network is a social structure consisting of individuals (or organizations) called nodes and ties that connect nodes by different types of interdependency, such as friendship, affiliation relationship, communication mode or relationship of religions, interest or prestige[21]. Social network graphs are constructed using information found in different places, such as the contact lists from social networking sites, the phonebook from mobile devices, and blog rolls. Social network graphs help to recognize how members overlap and be able to use information to synchronize the contacts on different social networks. This information can then be used to enable communication with the people found in the other social networks. There are many projects that try to help in creation of social networks graphs and to make this information available to anyone. Friend of a Friend (FOAF) ${ }^{1}$ and XHTML Friends Network $(\mathrm{XFN})^{2}$ are examples of these projects. The Friend of a Friend (FOAF) project aims at establishing a web of machine-readable pages describing people, the relationships with each other, and the things that they make and do. Unlike FOAF, XFN, is not actually a social network, but is a microformat used to characterize relationships between individuals using links in an efficient way. Both try to create a network of web pages that are easily interpreted by computers. These web pages contain information about relationships between people. Traversing those networks and collecting information about the

${ }^{1}$ FOAF. The Friend of a Friend (FOAF) project. Foaf. http://www.foaf-project.org/

${ }^{2}$ Global Multimedia Protocols Group. XFN - XHTML Friends Network. GMPG. http://gmpg.org/xfn/ relationships could be applied automale to tically. However, people may use different aliases in different social networks. The OpenID project ${ }^{3}$ tries to solve this problem of each person having several aliases across the web by giving each person a unique URI as ID. OpenID enables Internet users subscribe to many different web sites using a single digital identity, thus reducing the number of user names and passwords each person needs. It is a decentralized, free, and open standard that lets users control the type of information they want to provide. Another type of social sites is SyncML ${ }^{4}$ which is an open, platform independent, information synchronizing and device management standard for mobile devices. The development of SyncML was started by the SyncML initiative, but currently is consolidated into the Open Mobile Alliance (OMA). It is now supported by many mobile device manufacturers and SyncML clients are pre-installed in most mobile phones and devices. Another mobile social network was introduced by [9] that supported location-based, personalized, interactive mobile social network services using mainly metropolitan Wi-Fi networks in the context of social services. The research was oriented towards on the concept of mobile communications could be used to increase the closeness of one's social networks[8].

\subsection{Scientific social networks}

These social media are characterized by having traditional features of a social-networking with the addition of a daily science newsfeed, lab profiles, a science forum, blogs, etc. The main advantage of those sites is to provide space for researchers to create their own user profile, add their publications history, upload technical research protocols, and the ability to share research articles with the community. Furthermore, some of the sites are able to host a free video conferencing service to facilitate long distance collaborations with other science and journal clubs. Researcher network, a social network between researchers mainly based on coauthorship and citation relationship [10]. It focuses on helping researchers to discover research trends and performance of its members. It could be also used to indicate key researchers in a researcher group, and further to facilitate finding appropriate contact point for collaboration in an easy way [12]. Several researcher network services are currently on the Web. BiomedExperts ${ }^{5}$ shows co-publication between researchers and the researchers relating with a selected one in biomedical domain [24]. Research $\mathrm{GATE}^{6}$ by its name symbolizes the importance of efficient and result-driven search functionality within research in general and within the network in particular. It provides additional service function for grouping researchers by contacts, publications, and groups. Metadata of a researcher is also found as well for every node. Recently it applies ReFind search engine which is based on "intelligent" correlations. It enables the scientists to find groups, papers, fellow researchers and everything else within and outside of ResearchGATE. Next, ScienceStage.com - Science in the 21st century - appear as a wide forum for science that is based on

${ }^{3}$ The OpenID Foundation. OpenID » What is OpenID?. The OpenID Foundation. http://openid.net/what/

${ }^{4}$ Wikipedia. SyncML - Wikipedia the free encyclopedia. Wikipedia. Last modified 2008- 08-27. 8. http://en.wikipedia.org/wiki/Syncml.

\footnotetext{
${ }^{5}$ http://www.biomedexperts.com
}

${ }^{6} \mathrm{http}: / /$ www.researchgate.net 
an interdisciplinary, international and individual level. ScienceStage.com is now considered as one of the universal online portal for science, advanced teaching and academic research. It bridges a major gap in scientific research and learning. ScienceStage.com is a virtual conference room, lecture hall, laboratory, library and meeting venue all in one [13]. There are other well-known social media sites and networks for scientists that are listed here without any particular order:

- Nature Network - uber network from the publishing giant

- BioMedExperts - Scientific social networking

- BioWizard - Blogged up Pubmed search

- Mendeley - Digital paper repository and sharing

- Labmeeting - Ditto

- YourLabData - socialised LIMS

- SciLink - Sci-Linkedin

- Myexperiment.com - mostly workflows.

\subsection{Social network analysis}

Social network analysis (SNA) is a set of techniques used to study the exchange of resources among actors (i.e., individuals, groups, or organizations) [11]. It aims to reveal or visualize the relationships resided in the network using network theory. Basically the graph theory is a common principle that is used to conduct such analysis. Social network analysis is studied in social science areas such as psychology, sociology, behavior science and organization science discipline in several decades ago $[2,21,23]$. SNA has been an instrumental tool for researchers focusing on the interactions of groups since the concept was introduced by Moreno[15]. Social networks decompose interaction behaviors between various individuals from the different aspects, this would affect the traditional analysis of that networks. Since the graph-based structure is often very complex. Sociograms were considered a formal representation of the patterns of interpersonal relationships upon which larger social aggregates. Applying this representation, graphs are used to represent relationships between individuals in a group or community. Graphs, or sociograms, were created to analysis the interactions of some communities such as New England communities to study their social and political relationships to detect such relationship between individuals in a group or community. The extension of the sociogram concept into group dynamics occurred in combination with the concept that individuals or organizations exchange information during the performance of any activity [10,23]. Given the premise that any activity requires a transfer of information and knowledge, the extension of this foundation is that these exchanges can be mapped within sociograms where actors and information exchange become nodes and arcs within the graph [24]. The ability to apply mathematical analysis to network information exchange provides researchers with established measurements for analyzing the effectiveness and weaknesses of the group being studied [1]. Another approach was introduced [17] to add constraints during clustering process in order to improve the bonding and bridging ion side and between groups of similar interest and therefore helps to predict important outcomes and trusted community However, our proposed system applies similar linkage like sociogram to group actors of social network using ontology which is used to define relationships among them.

\section{UTILIZING SOCIAL NETWORK FOR INFORMATION SHARING}

Information sharing involves the transfer or distribution of information from one person or group to another. World Wide Web (WWW) is used for information sharing between people or organizations. The proposed framework utilizes social network to enhance collaborative work between people work on the same domain in order to deliver the right knowledge to the right person (groups) based on their role, preference and interest. User modeling includes static information (explicit) which is delivered by user as well as other dynamic information (implicit) which is extracted based on user interactions. Accordingly, the system would be able to categorize users into groups according to their preference and interest. This section discusses the role of ontology in modeling of users which is the core of the knowledge sharing environment.

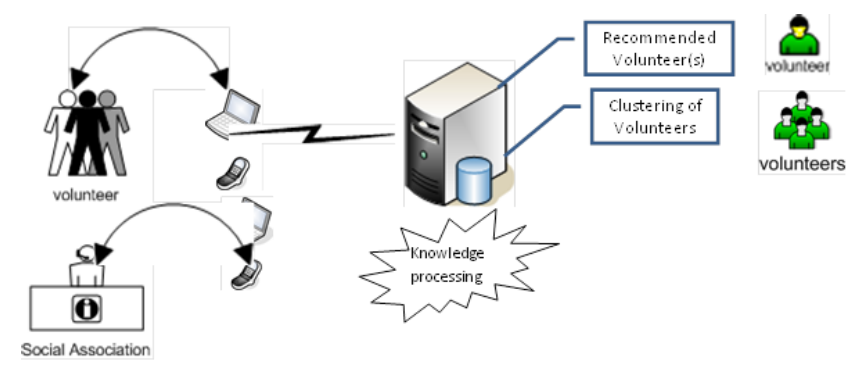

Figure1: Framework of the society in hand system

\subsection{Modeling of user's profile}

Knowledge modeling is the conceptualization and representation of problem-solving knowledge in a knowledge base. In the proposed framework, user model is built using both explicit as well as implicit information. Currently, society in hand has two distinguished categories of users: the first category includes social associations who represent the organizational part of community that is capable to deliver social services. The second category of users is the volunteers who are individuals willing to do social and community services but are not aware about it. As shown in figure1, users supply their details through friendly web interface. This information includes personal details such as name, address, gender, email, as well as a set of community services that each party could deliver to community. This type of information represents the static identity of each user. While usage data is extracted to express the influence of the users through her/his real interaction. For example, volunteers interact in order to: accept community service to apply, post information and discussion related to community work, send message to other members. While, social associations interact with the system by requesting a set of community services to be applied, posting information and discussion related to community work, sending message to other members. They also have the ability to rate the work applied by each volunteer based on their excellency in doing the assigned community service. This behavioral information represents the semantic part that is included in user's profile which is a real measurement of the contribution and effectiveness of the user. Adding semantic to profiles of the user is a key to measure their tendency to apply the same community service are therefore cluster volunteers into groups based on their interest. Ontology is used here to semantically relate users to each other's w.r.t annotated services. This section discusses 
the role of ontology to extract community of similar people based on their interest.

\subsection{Matching ontological profiles}

Currently, social networking allows users to proactively connect to each other. The user is not considered an isolated individual any more, but a member of one or more communities [4]. System such as developed in [19, 26] assumed that social relationship could exist through participating in the same events or activities involving two or more individuals. Such events might not require personal contact, but they must involve social interaction. Those systems were able to retrieve hidden links between individual based on their interaction to construct community of practice in specific domain. While other system [18], built trust seeds for each individual within the social network by considering the strength of users' relationships to identify a group of trust. Combining these approaches, in society in hand, members are grouped based on their conmen interest which is domain dependent and expresses their bonding to specific community service(s) and stored in their ontological profile. Profile of each user is constructed by a combination of two models:

- Stated Preferences: This model scores users according to how closely they match their stated information (explicitly defined by user) such as: address and selected community services. Each of these features is initially defined by each volunteer and therefore they are considering having a static affects the clustering process.

- Learned Preferences: This model works similarly to the stated preferences, but learns from observed user's behavior. Semantic features are used to dynamically related volunteers such as their tendency to post and discuss topics that are related to the same community service(s), their ability to accept to apply the same type of community service from different social association as well as the accumulated rank assigned by different social associations. This provides an indication about her/his ability to do this type of service and as a result volunteers who have similar ranks are grouped together. Users with similar profiles are grouped together based on their closeness which is measured using the semantic relations between concepts stored in the ontology.

\subsection{Society-in hand ontology}

In its classical sense, ontology is a philosophical discipline, a branch of philosophy that deals with the nature and the organization of being. In its most prevalent, use an ontology refers to an engineering artifact, describing a formal, shared conceptualization of a particular domain of interest [7]. Ontology contains classes, properties and relationships that are used to encapsulate existing knowledge about a specific domain. Nowadays, Ontology can be used to attenuate problems generated by information heterogeneity and to construct virtual community based on the representation of a member's profile containing personal, behavior, and professional information As shown in figure 2, the domain concepts are organized as a hierarchical structure. Society-inhand (SIH) ontology contains four main concepts: Volunteers, Charity, Discussion, and Services These abstract concepts provide a wide and flexible range for the capture of knowledge about community field. The concept sih:Volunteer can be thought of as the central point for the ontology. A volunteer begins by describing himself or herself, listing key identity attributes

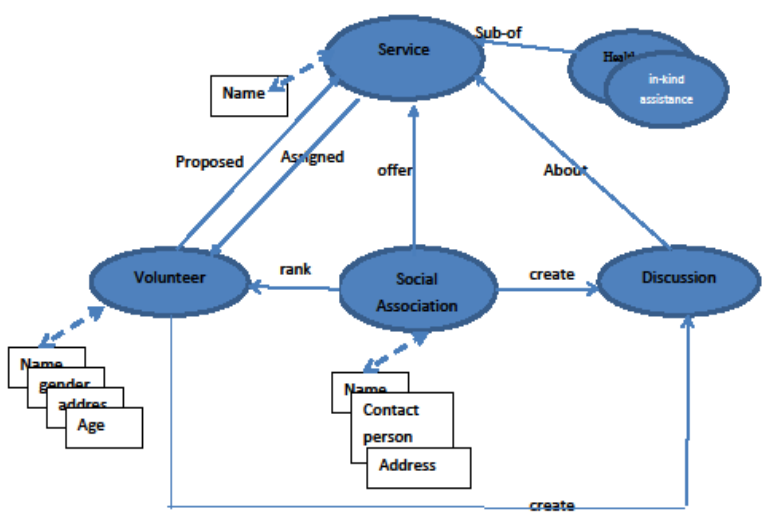

Figure2: Structure of society in hand ontology

such as name, gender, age and address. It also contains proposed-services attribute that contains a list of services concept/sub concept names that the volunteer can offer. This information is set during signing up. Volunteer concept also has assigned-services relationship with service. The assignedservices relationship contains information regarding the following attributes: status, rank and frequency. Status attribute may have one of three values represent the status of the service (complete, in progress, canceled). The rank attribute contains based on the charity(s) feedback upon completion of that service and is used to increase the score of the volunteer. The last attribute is the frequency which contains the number of times that the volunteer applied this service. The concept sih:charity is described by the following attributes: charity-name, address, contact person, email, phone number as well as the set of services that charity offer. It also contains relationship called has_charity with volunteer to represent relationship between them. The concept sih:service declares all available community services such as: as in-kind assistance, health care services, provide health care and the full nutritional, care for orphans, street children, care of handicapped, special needs, etc. The concept sih:discussion is used to decompose post and discussion information. As the system allows volunteers/and charities to interact and communicate with each other through discussion board. It has relations create with both charity and volunteer concept that is used to decompose post and discussion information related to each instance of service concept.

\section{SYSTEM ARCHITECTURE}

The architecture of Society-in-Hand system follows the 3-tier architecture that has two main elements the front end and the back end. The front end, client side, allows users to interact with the system. The backend (server side) contain all possible information that is needed for the users of the system. Figure 3 shows the proposed architecture which will be described in the following subsections.

\subsection{Data representation layer}

Society in hand allows its users (volunteers and charities) to supply their basic information and to post upcoming information such as posting dweller cases, urgent requested service, etc. This data would be stored in the common representation forms in order to be processed and compute interdependencies between knowledge items. Then it would be used in clustering of candidate volunteers and services.

\subsection{Knowledge sharing Layer}

Knowledge sharing is an activity through which knowledge (i.e. information, skills, or expertise) is exchanged among people. Knowledge sharing involves the transfer of knowledge from one person or group to another. Knowledge 
sharing layer is the core of the proposed system since it allows stakeholders of the system to identify themselves, publish their activity, communicate with each other, etc. Therefore, this layer is decomposed into three main components: profile manager, which allows system users to define themselves, the other component is the community extraction module which extracts the sematic features of the users. The third is the ontology-based clustering engine, which is used to divide volunteers into groups based on their semantic similarity. In the following details description of each component is provided.

\subsubsection{Profile manager}

The main function of the profile manager is to allow users of the system to access and update their information. The user of the system should be able to define her/ himself by supplying personal information such as, name, qualification, email, address, gender. Volunteers and charities also identify the list of services they are capable of doing in details. Furthermore, the user has the ability to update her/his information at any time.

\subsubsection{Community extraction}

Our approach to extract community of volunteers is based on modeling of profiles of users with reference to domain ontology and matching their profiles. In social networks, connection between members that occur virtually appears in form of sharing preferences, items and knowledge. This would lead to get benefit from each other's experience in specialized field of interest, even if they have different profiles as a whole. Therefore, these preferences and interests could be extracted and measured with reference to interrelated domain concepts. Profiles of users are initially described as weighted lists of domain concepts taking advantage of the relations between concepts, and the (weighted) preferences of users for those concepts. Then, the system clusters users based on the correlation of concepts appearing in their profile. In society in hand, we tie individuals (volunteers) to each other based on their tendency to do a specific community service(s) i.e. to service concept as will be illustrated in the next section. Our approach assign a value for each static and learned preference for each service concept to be used for similarity measurement. Within the overall similarity computation approaches, there are three distinguished dimensions [13]:

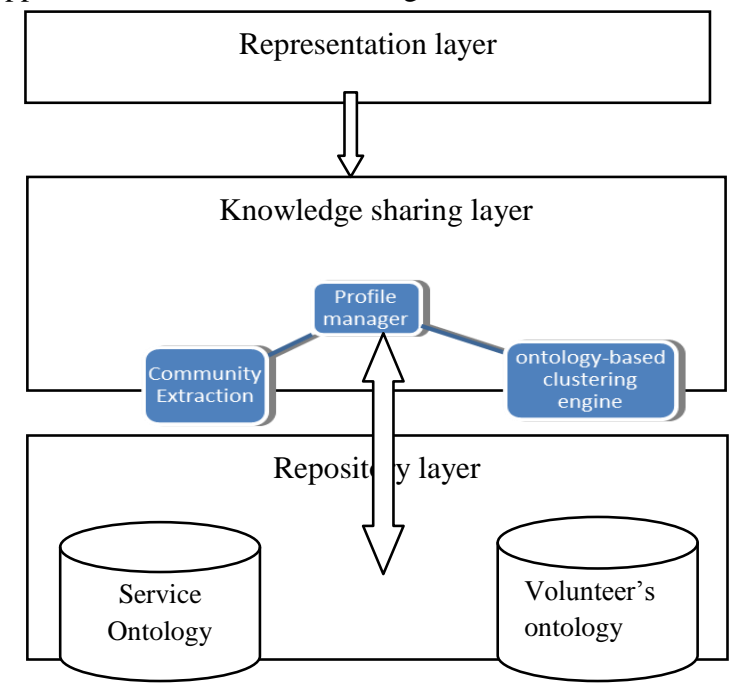

Figure3 Society-in-Hand system architecture
- Taxonomy similarity: Computes the similarity between two instances on the basis of their corresponding concepts and their position in ontology.

- Relation similarity: Compute the similarity between two instances on the basis of their relations to other objects.

- Attribute similarity: Computes the similarity between two instances on the basis of their attributes and attribute values.

Our proposed similarity measurement technique is based on relation similarity which assumes that it is possible to develop methods capable of detecting similarities between conceptually similar objects even when they don't contain lexically similar terms provided that they have common relations with other object within the same domain. Therefore, volunteer who has common interest in a specific concept (in our case service community concept) are assumed to be connected in the social network, and their preference weights related to that concept is the key measure of the degree of membership to that cluster.

\subsubsection{Ontology-based clustering engine}

Clustering is used to group persons inside a community with similar interests. Users in the same group are held together by a common interest in a body of knowledge, and are driven by a need to share problems, experiences, and best practices [13]. Recently, matching and clustering are used to calculate the closeness between web items based on the degree of similarity between different web resources [20]. Hierarchical agglomerative cluster [5] analysis is widely applied for assemblage studies to create a hierarchy of clusters. The proposed clustering engine starts by calculating the correlation coefficient for each identified features in the user profile. This coefficient is used to measure the closeness between volunteers and then apply clustering technique. Then, it treats each volunteer as a singleton cluster, and then other volunteers are successively joined based on similarity until all volunteers are distributed over remaining clusters. A tree structure called a dendrogram [5] that visually depicts this hierarchal sequence. The process of clustering is described in details in the following section

\subsection{Repository layer}

The main goal of the repository layer is to acts as a destination hub for individual and organization profiles to establish relationships with co-workers. Thus, community of users is able to jointly build, or expand, their social networks. It contains all data about the following: volunteer data, social association data, and service ontology.

\section{ONTOLOGY BASED CLUSTERING PROCESS}

This section describes how the clustering process is applied based on measuring the correlation coefficient between volunteers

\subsection{Measuring Similarity between users}

Clustering of requires some kind of similarity measurement that is computed based on the semantic of ontology. Preferences of each user are represented as a vector of objects for each service with its associate weights (numbers from 0 to 1) [3]. The user preferences depend on the value of attributes 
(features) of the objects, The values associated in each field reflect the intensity of the user 's interest for that feature such that the lower the value, the measure of dislike for that feature. This is accomplished through two steps, the first one is to define the features that constitute the service vector, and then calculate correlation coefficient for each feature.

\subsubsection{Identify domain specific features}

The similarity measurement technique applied here is based on relation similarity that indicates that the similarity of two instances depends on the similarity of the instances they have relations to. Therefore, two volunteers are similar based on their relationship to each community service. As a result, we calculate this relationship as an aggregation of two main types of features:

(1) Explicit well-defined relationships and,

(2) Semantic inferred relationships

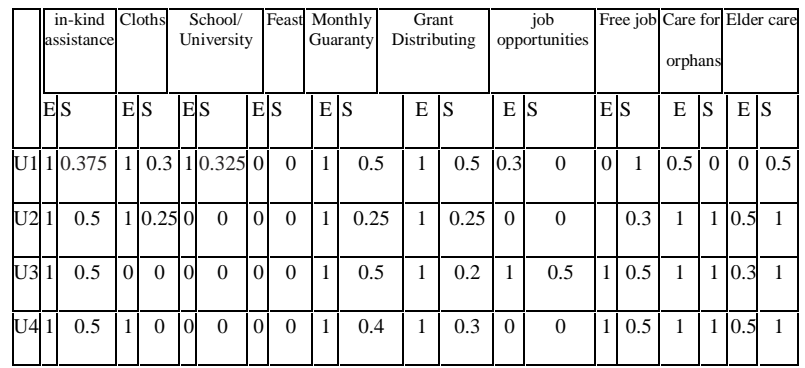

\section{Table2: Closeness metric of User vectors}

Explicit well-defined relationship is used to group volunteers who show interest to provide the same voluntary work since This value is binary to indicate whether the volunteer offer to apply this service (1) or not (0). While semantic value (S) is used to indicate the affinity of the volunteer towards that service and is calculated as an average of the following items, the frequency of user post a discussion about this service, the number of times the user had previously apply this service, and the trust score accumulated from social association he deals with. Table1 represents how the profile of the volunteer is used to include the assigned services with its associate features.

\begin{tabular}{|c|c|c|c|c|c|c|}
\hline service & Expli & \multicolumn{5}{|c|}{ Semantic } \\
\hline fegture & 0ffered & post & discuss & frequency & Trust & total \\
\hline $\begin{array}{l}\text { in-kind } \\
\text { assistance }\end{array}$ & 1 & 0.5 & 0.2 & 0.4 & 0.4 & 0.375 \\
\hline $\begin{array}{l}\text { School/ } \\
\text { University } \\
\text { payment }\end{array}$ & 1 & 0.3 & 0.2 & 0.5 & 0.3 & 0.325 \\
\hline Cloths & 1 & 0.2 & 0.6 & 0.1 & 0.3 & 0.3 \\
\hline
\end{tabular}

Table1: Example Profile of Volunteer1

The information stored in that table indicates that volunteer1 offered the following services: in-kind assistance, school payment, and providing clothes with frequency of $40 \%$ inkind assistance, with 50\% School/ University payment, with $10 \%$ cloths. Furthermore, volunteer 1 obtained accumulated trust score from different association with the following rate, $0.6,0.8$ and 0.2 for in-kind assistance, school/ university payment, and cloths respectively. Volunteer1 provides posts about each of the assigned service with the following ratio $0.5,0.3$ and 0.2 and share in discussion with a percentage of $0.2,0.2$ and 0.6 respectively. It is significant to mention that we use the average to calculate the value for each individual feature. i.e, if volunteer1 posts in 5 different discussions, according to table1, it means that he shared in 3 regarding inkind assistance and 1 related to school/ university payment, and one for cloths. Finally, the value of semantic inferred relationships is calculated as an average for all that features.

\subsubsection{Similarity Computation}

Each volunteers is represented as a vector of services $\mathrm{Vj}=\left[\mathrm{Sj}_{\mathrm{j}}\right.$, $\mathrm{Sj} 2, \mathrm{Sj} 3, \mathrm{~S} \mathrm{j4}, \ldots . . . \mathrm{SjM}]$ where each service is represented with two values explicit and implicit that indicate the intensity of user $j$ to each of his proposed service. Finally, all users are collected in the closeness matrix $\mathrm{M}$ as shown in table2. Based on this matrix, classic hierarchical clustering algorithm [22] is applied which assumes that if items in the same cluster are closed as they have very similar distances to all other entities in the same group, they must be very similar. Over the years, many prominent ways have been used to compute the similarity. The most commonly used distance functions for clustering algorithm are the Euclidean distance, Manhattan (city block) distance and Cosine correlation measure. The commonly used similarity measure when applying refereeing to ontology in clustering is the cosine correlation measure [25], given by:

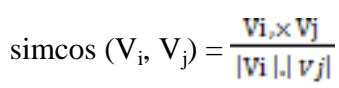

\subsection{The Clustering process}

Society in hand architecture aims to construct group of volunteers based on their semantic similarity. Correlation coefficient for each of the identified features is estimated with reference to the ontology. The clustering process applies the following steps:

Step1:

Create a vector $\mathrm{V}_{\mathrm{i}}$ correspond to each volunteer and contains stated and learned preference

$V_{i}=[\mathrm{Sj} 1, \mathrm{Sj} 2, \mathrm{Sj} 3, \mathrm{~S} \mathrm{j} 4, \ldots \ldots . \mathrm{SjM}]$

where $\operatorname{sj} \epsilon[0 \ldots \ldots$ l 1$]$

Step 2:

Create a cluster $\mathrm{C}_{\mathrm{i}}$ correspond to each volunteer

Step3:

Calculate the correlation coefficient for every stated and learned preferences.

Step4:

Merging of the two volunteers $\mathrm{V}_{\mathrm{i}}$ and $\mathrm{V}_{\mathrm{j}}$ having the highest similarity values in a closeness matrix $\mathrm{M}$

Step5:

Whenever a newly defined volunteer is added, similarity is calculated between the newly defined and each remaining cluster $C_{n}$ in $M$. which is used to update the row and the column of $\mathrm{M}$ related to the newly defined volunteer 


\section{SOCIETY IN HAND}

Society in hand has been implemented using java (J2EE) integrated with MySQL database to implement the backend while css, javascript and Yui tool are used to implement the front end. Society in hand is available in both English and Arabic language. Unlike other social network such as jumo.com which only join people with nonprofits and charitable organizations without any suggestion about the type of work that they are capable of doing it based on their experience in applying that work. Furthermore, society in hand provides a specific feature, which is allowing charity to define two types of services: ordinary service and emergency services. Ordinary services are the services that a charity used to perform while emergency services are defined as the services that are needed to be applied in an urgent manner so that volunteers are notified either through email. It also provides its two types of activities that target either individual or community.

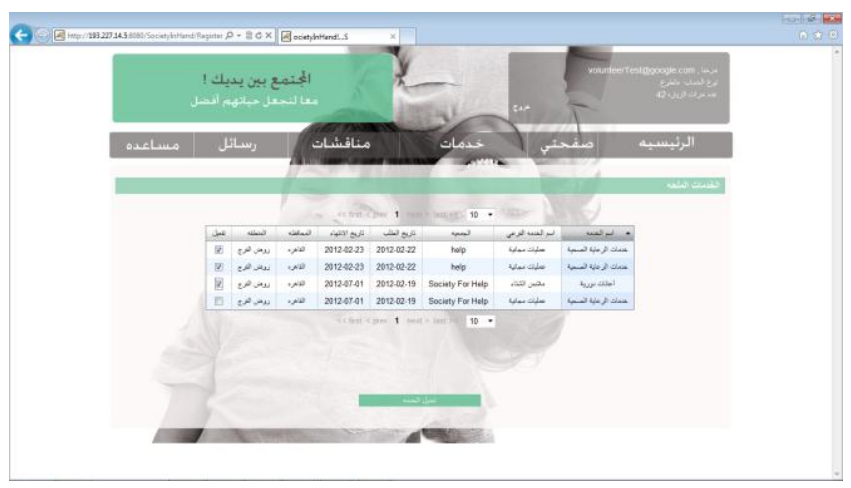

Figure4: Snapshoot of society in hand system

and target specific system user based on user context. These services include: notification about available community service(s), suggestion of friends. While community services involve services that are delivered to a community of volunteers such as notification about urgent community service(s) and discussion about a specific issue. Community activities are applied based on the clustering process mentioned above. Furthermore, society in hand provides its users the facility to generate a set of reports regarding their activities such as: Listing current available services, viewing charity to volunteers recent services, send/receive message. Figure 4 represents a snapshot for the system that shows the assigned service to a specific volunteer based on her semantic profile.

\section{CONCLUSION AND FUTURE WORK}

This paper describes a framework for facilitating the communication and connection between individual who work in community services field. It targets utilizing social network to help dwellers with adequate services that would help to face their daily challenge. Our approach is oriented to utilize social network by offering a web environment through which different entities such as volunteers and social associations could interact to provide adequate service for those who need it. The basic idea is to help social association and charity which are sometimes not able to serve the huge number of dweller due to limitation in its resources with several volunteers who intended to provide their help in different domains such as (health care, education, etc). Therefore, the proposed system would connect those parties with each other's in order to aggregate the overall activities to help poor people. Society in hand is an attempt to provide the awareness of the "social duties towards slum's dwellers" to the community in an easy, efficient and rapid way. An ontology based similarity measurement technique was developed in order to cluster volunteers into groups who share the same interest. The user of the system would communicate with each other through a social network which enables them to interact and post their activities. The system is currently extended to enable user to interact through mobile device. Currently, the field of cloud computing has developed options that allow capturing different types of information using social networking. Therefore, we aim to extend this work and utilize cloud computing technology to extract more knowledge about the users of the systems that enrich the similarity measurement features used here. Also, we intended to add some location based features such as allowing volunteer to interact with each other according to their current location.

\section{ACKNOWLEDGMENTS}

This paper is developed from the Winning Research Competition Granted by "CU/UNDP MDGs Awareness \& Research Initiative" within the framework of Cairo University (Community Service \& Environmental Development Sector) during 2010-2012.

\section{REFERENCES}

[1] Alba, Richard D. (1982). Taking stock of network analysis: A decade's results. Research in the Sociology of Organizations, 1, 39-74.

[2] Barnes, J. Social Networks Reading. MA: AddisonWesley, 1972.

[3] Castells, P., Fernández, M., Vallet, D., Mylonas, P., and Avrithis, Y. (2005). Self-Tuning Personalised Information Retrieval in an Ontology-Based Framework. Proceedings of the $1^{\text {st }}$ International Workshop on Web Semantics (SWWS 2005), Agia Napa, Cyprus. Springer Verlag Lecture Notes in Computer Science, vol. 3762, pp. 977-986.

[4] Georgios Paliouras. "Discovery of Web user communities and their role in personalization", User Modeling and User-Adapted Interaction, Vol 22, no 1-2 pp:151-175 , 2012

[5] Dubes, R. C. and Jain, A. K., Algorithms for Clustering Data, Prentice Hall (1988).

[6] Goodstein, A. (2007). Totally wired: What teens and tweens are really doing online. New York: St. Martins Griffin

[7] Gruber, T. R. A translation approach to portable ontology specifications. Knowledge Acquisition, 6(2):199-221, 1993.

[8] Han, J. and Kamber, M. 2006. Data Mining: Concepts and Techniques (2nd edition). Morgan Kaufmann.

[9] Haw-Yun Shin. "A General Architecture of Mobile Social Network Services", 2007, International Conference on Convergence Information Technology (ICCIT 2007), 0/2007

[10] Hanmin Jung, Mikyoung Lee, Pyung Kim and Seungwoo Lee, "Generating Researcher Networks with Identified Persons on a Semantic Service Platform", Recent Trends 
and Developments in Social Software, Lecture Notes in Computer Science, 2011. 978-3-642-16580-1

[11] Haythornwaite, C. (1996). Social network analysis: an approach and technique for the study of information exchange. Library and Information Science Research, 18(4), 323-342.

[12] Jothi P. Sri \&. M. Neelamalar, The Study of Social Media Communication: Analysis of Science Communication through Social Networking Sites with special reference to Scientists, Estudos em Comunicação no8, 1-11 DEcember 2010.

[13] Maedche, A. and Zacharias, V. 2002. Clustering Ontology- Based Metadata in the Semantic Web. Lecture Notes In Computer Science. Proceedings of the 6th European Conference on Principles of Data Mining and Knowledge Discovery. Springer-Verlag London. Vol. 2431, 348-360.

[14] Mehul Motani, Vikram Srinivasan, and Pavan S. Nuggehalli. 2005. PeopleNet: engineering a wireless virtual social network. In Proceedings of the 11th annual international conference on Mobile computing and networking (MobiCom '05). ACM, New York, NY, USA, 243-257.

[15] Moreno, Jacob L. (1960). The Sociometry Reader, Glencoe, IL: The Free Press.

[16] Pronovost, S., Lai, G.: Virtual Social Networking and Interoperability in the Canadian Forces Netcentric Environment. Technical report CR 2009-090, Defence R\&D Canada (2009)

[17] Rajkumar, J and Narendra S Representation of K-Cluster Constraint as K-Sat in Social Networking. IJCA Special Issue on Evolution in Networks and Computer Communications (1):13-18, 2011

[18] Samah Al-Oufi, Heung-Nam Kim, Abdulmotaleb El Saddik, A Group Trust Metric for Identifying People of
Trust in Online Social Networks, expert system with application , June, 2012

[19] Silva, J.L.T. ; RIBEIRO, A. M. ; BOFF, E. ; PRIMO, T.T ; VICARI, R. M. . A Reference Ontology for Profile Representation in Communities of Practice. In: Metadata and Semantics Research Conference, 2011. Fifth Metadata and Semantics Research Conference (MTSR 2011)

[20] Silvana Castano, Alfio Ferrara, and Stefano Montanelli. 2012. Structured data clouding across multiple webs. Inf. Syst. 37, 4 , 352-371.

[21] Scott, John (1991). Social network Analysis: A Handbook, Sage: London.

[22] Ungar, L., Foster, D.: Clustering Methods for Collaborative Filtering. Proceedings of the Workshop on Recommendation Systems at the 15th National Conference on Artificial Intelligence, AAAI Press, 1998.

[23] Wasserman, Stanley, \& Faust, Katherine. (1994). Social network analysis. Cambridge, MA: Cambridge University Press.

[24] Whitaker, I., Shokrollahi, K.: BiomedExperts: Unlocking the Potential of the Internet to Advance Collaborative Research in Plastic and Reconstructive Surgery. J. Annals of Plastic Surgery 63(2) (2009)

[25] Wiesman, F. and Roos, N. Domain independent learning of ontology mappings. In AAMAS, pages 846-853, 2004.

[26] Yutaka, M. "Real-world oriented information sharing using social networks", Proceedings of the international ACM SIGGROUP conference on Supporting group work - GROUP 05 GROUP 05, 2005 